\title{
Soziale Versorgung in ökonomischer Verantwortung
}

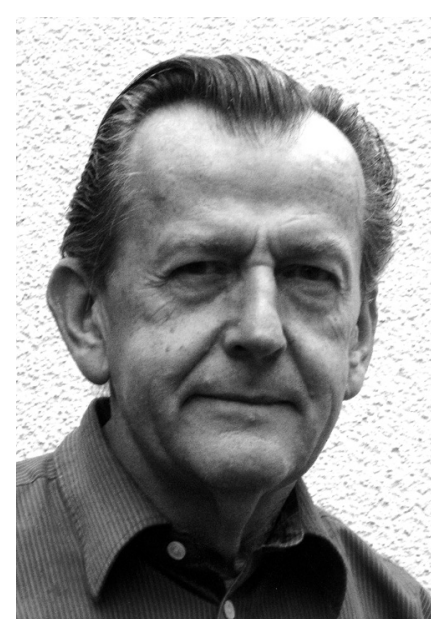

VON WOLF RAINER WENDT Prof. Dr. Wolf Rainer Wendt ist Vorsitzender der Deutschen Gesellschaft für Care und Case Management sowie Honorarprofessor der Eberhard Karls Universität Tübingen. Er ist Herausgeber der Buchreihe »Forschung und Entwicklung in der Sozialwirtschaft" in der Nomos Verlagsgesellschaft in Baden-Baden und Mitglied im Beirat der Zeitschrift SOZIALwirtschaft und des Informationsdienstes SOZIALwirtschaft aktuell. prof.dr.wendt@googlemail.com

\author{
Viele Aufgaben im Sozialleistungssystem \\ könnten erwerbswirtschaftlich erfüllt werden; \\ aber bei dem oft zufälligen Zustandekommens \\ des Angebots in einem freien Markt, ließe sich \\ damit gesellschaftliche keine umfassende und \\ verantwortliche Daseinsvorsorge gründen.
}

Einrichtungen und Dienste der Wohlfahrtspflege betreiben kein unabhängiges Geschäft. Sie können die Preise für viele ihrer Leistungen nicht festlegen und ihr Angebot unterliegt Regulierungen, die auch über die Nachfrage bestimmen.

Sozialunternehmen erfüllen einen gesellschaftlich ausgemachten Versorgungsauftrag, auch wenn sie ihn in eigener Mission für sich reklamieren oder mit einem innovativen Angebot erreichen, dass sie im Rahmen eines damit erkannten Bedarfs beauftragt werden, ihn zu decken. Die Einbindung der einzelnen Unternehmungen in das soziale und gesundheitsbezogene Versorgungsgeschehen lässt sich, wie im Folgenden argumentiert wird, positiv auffassen: Als ein dynamischer Komplex erfüllt die Wohlfahrtspflege insgesamt ihre sozialwirtschaftliche Funktion.

Die freigemeinnützige Wohlfahrtspflege hat sich im vorigen Jahrhundert im Wechselverhältnis von Staat und selbständig organisierter Wohltätigkeit entwickelt, wie jüngst ausführlich dargelegt wurde (vgl. Kasten). Ihre Institutionalisierung geschah in Kriegs- und Nachkriegszeiten; sie wurde gebraucht - und sie bekam für ihren Einsatz die öffentlichen Zuwendungen, die heute im europäischen Binnenmarkt mit seinem Wettbewerbsund Beihilferecht Anstoß erregen.

Der wohlfahrtsstaatliche Versorgungsauftrag, der den Freien Trägern gegeben wurde und den sie weiterhin in Kooperation mit der öffentlichen Wohlfahrtspflege erfüllen, schließt nach dem Subsidiaritätsprinzip jene Zuwendungen ein, ohne dass sie damit eine Begünstigung darstellen. Die Gewährleistung des Staates in der Daseinsvorsorge baut auf die andauernde Bereitstellung der nötigen Dienste und Einrichtungen seitens der freigemeinnützigen Wohlfahrtspflege insgesamt - unabhängig vom Angebot privater Dienstleister, die im übrigen für einzelne Leistungen, die sie beibringen, die gleiche Vergütung wie die wohlfahrtsverbandlich organisierten Anbieter erhalten.

Die freigemeinnützigen Träger nehmen intermediär eine Infrastrukturverantwortung wahr, die auch für das Geschäft der beteiligten Sozialunternehmen getragen wird. Auf die Dynamik des örtlichen oder regionalen Bedarfs kann zu dessen Bewirtschaftung laufend mit einer Diversifizierung des Leistungsangebotes reagiert werden. Die Partnerschaft der Verbände und Ligen mit der öffentlichen Hand erlaubt Beweglichkeit in der Gestaltung der Versorgung.

Das Auftragsverhältnis ist in der Beziehung von Staat oder kommunaler Gebietskörperschaft auf die Freier Wohlfahrtspflege nicht von gleicher Natur wie in den Versorgungsbereichen Energie, Wasser oder Telekommunikation, in denen die Privatisierung fortgeschritten ist. Der konkrete Einsatz von Menschen für Menschen bleibt in der Vielseitigkeit, in der die Humandienste benötigt werden, auf ständige und im Raum des Zusammenlebens verteilte Bereitschaften angewiesen.

Als Mitgliederorganisationen halten die freigemeinnützigen Verbände diese Bereitschaften vor. Sie kommen zur Be- 
wältigung diverser sozialer Herausforderungen, wie es sie etwa in der Jugendhilfe gibt, und von Problemlagen wie der Versorgung von Demenzkranken zum Einsatz. Verlangt wird keine gleichmäBige Bedienung der Bevölkerung wie im Falle von Strom und Wasser, sondern eine personenbezogen differenzierte Versorgung.

Im sozialen Haushalt, welcher der Daseinsvorsorge gewidmet ist, wird bei personenbezogenen Dienstleistungen mit den vorhandenen Strukturen gerechnet. Neue mögen hinzukommen oder sie ablösen, aber angesichts des komplexen und dynamischen Bedarfs kann in der Versorgung nicht darauf gewartet werden, dass sich im Markt das passende Angebot einstellt.

Die vermittelnden Instanzen der Wohlfahrtspflege sorgen für Arrangements, die auch bei Wandel und Austausch von Elementen stets zur Verfügung stehen. Im Gegenzug schaffen Ansätze in kommunalen und staatlichen Haushalten zur Förderung der Freien Wohlfahrtspflege für sie Planungssicherheit und tragen so zur Gewährleistung einer andauernden und gleichmäßigen Versorgung bei.

Der Versorgungsauftrag wird von der Wohlfahrtspflege in corpore wahrgenommen, von ihren Diensten und Einrichtungen beieinander im Gewebe ihrer Verbände. Die Erfüllung des Auftrags erfolgt, bei allen Mängeln und Schwierigkeiten, im ganzen und hängt nicht an der Vergabe an das eine oder andere Sozialunternehmen im Rahmen von Leistungsvereinbarungen. Deshalb ist eine Identifikation der verbandlichen Wohlfahrtspflege mit Interessen einzelner Unternehmen, die zu ihr gehören, nicht angebracht. Das einzelne Sozialunternehmen in der Wohlfahrtspflege steht im Anbieterwettbewerb mit Konkurrenten, die vergleichbare Leistungen erbringen. Es hat auf besondere Zuwendung(en) keinen Anspruch und man kann überhaupt fragen, ob in Hinblick auf seine Aufgabenerfüllung ein Unterschied zu privatgewerblichen Unternehmen besteht.

Es ist ohne weiteres vorstellbar, dass jede einzelne Aufgabe im Sozialleistungssystem im freien Markt übernommen und erwerbswirtschaftlich erfüllt wird. Aber mit der Kontingenz des Angebots, auf die sich der Gewährleistungsstaat dann einzustellen hätte, lässt sich in der Daseinsvorsorge nicht haushalten. In Ländern mit einem anderen Wohlfahrtsregime als dem hiesigen verlässt man sich nirgends auf kommerzielle Ausfallbürgen. Die Alternativen sind wieder sozialwirtschaftlicher Art wie in den romanischen Ländern, die auf die vie associative und genossenschaftliche Lösungen bauen.

Es hieße das Kind der Sozialordnung mit dem markt- und betriebswirtschaftlichen Bade ausschütten, meinte man mit einer Gleichstellung aller Anbieter von Humandiensten auch die freigemeinnützige Organisation der Wohlfahrtspflege erübrigen zu können. Sie vermittelt und organisiert in der Zivilgesellschaft wertegebundenes Engagement und bezieht in großem Ausmaß gemeinschaftliches Versorgungshandeln ein. Von ehrenamtlicher Mithilfe über Selbsthilfegruppen reicht dieses Handeln bis in die Eigensorge von Menschen, die sich zu ihrer Unterstützung bei der einen oder anderen Wohlfahrtsorganisation gut aufgehoben fühlen. Im Sozialraum ist es das Spektrum dieser Organisationen, mit dem die Diversität der Bedarfe bedient wird.

Die gewünschte sektorübergreifende Versorgung, die den komplexen Anforderungen gerecht werden kann, braucht, wenn sie denn im Sozialraum und damit nah an den sorgenden Bürgern erfolgen soll, ein breites Fundament. In der Freien Wohlfahrtspflege ist es als institutionalisiertes Engagement ausgebildet, erwachsen aus Initiativen von Einzelpersonen und von Gruppen. Die professionell Beschäftigten bewegen sich in einem Umfeld zivilgesellschaftlicher Beteiligung und sind in einer »caring community « auf informelle Mitwirkung angewiesen. Es besteht eine Abhängigkeit von den Individualhaushalten der Helfenden wie der Betroffenen, die zu ihrer Versorgung selbstaktiv beitragen und die Verantwortung für sie teilen. "Gemischte Wohlfahrtsproduktion " bringt eine Wechselseitigkeit des Faktoreinsatzes mit sich, auf die sich Wohlfahrtspflege künftig mehr und besser verstehen muss.

Die Ökonomie der Wohlfahrtspflege baut somit auf Ressourcen jenseits des Geschäfts eines einzelnen Sozialunternehmens. Sie ist auf das Netzwerk von Akteuren und auf ihre Aktivitäten verteilt, erbringt mit ihnen die Versorgungsleistungen nach sozialrechtlichen Maßgaben und verwendet darauf die

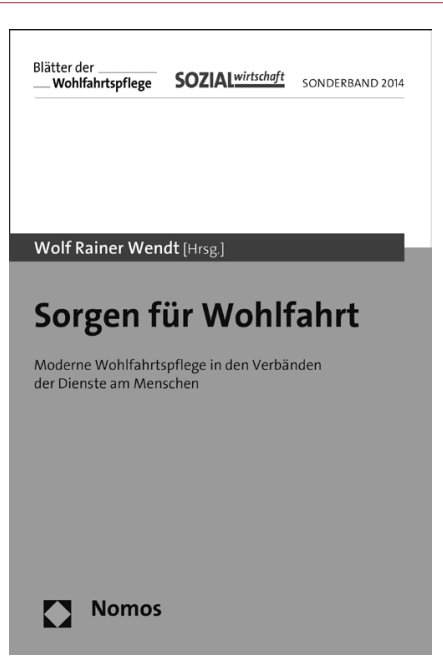

Die Freie Wohlfahrtspflege leistet in Deutschland einen Großteil der Versorgung hilfebedürftiger Menschen und beschäftigt mehrere Millionen hauptamtlich und freiwillig Mitarbeitende. Was aber Wohlfahrt und ihre Pflege eigentlich bedeuten und wofür die Verbände der Wohlfahrtspflege einstehen, bleibt gewöhnlich im Unbestimmten oder wird missverstanden. Nach vielen Jahren institutioneller Ausprägung der öffentlichen und freien Wohlfahrtspflege und ebenso langer Kritik an ihren Strukturen erfolgt in diesem Buch eine Verständigung über Wesen und Wandel, Stellung und Funktion der freigemeinnützigen Verbände. Sie sind sozialwirtschaftlich unterwegs in Gestaltung sozialer Daseinsvorsorge. Mit Beiträgen von Georg Cremer, Michael Löher, Rolf Rosenbrock, Uwe Schwarzer, Wolfgang Stadler, Gerhard Timm und Wolf Rainer Wendt.

Wolf Rainer Wendt (Hg.): Sorgen für Wohlfahrt. Moderne Wohlfahrtspflege in den Verbänden der Dienste am Menschen. Sonderband der Zeitschriften Blätter der Wohlfahrtspflege und SOZIALwirtschaft anlässlich des Jubiläums 90 Jahre Bundesarbeitsgemeinschaft der Freien Wohlfahrtspflege. Nomos Verlagsgesellschaft, Baden-Baden 2014. 180 Seiten. 39,- Euro. ISBN 978-3-8487-1195-6.

Zuwendungen aus dem öffentlichen Haushalt. Die Geschäftsführung des einzelnen Unternehmens, die Mühe hat, den laufenden Betrieb zu finanzieren, muss das ganze Aufgabenspektrum und die Ökonomie seiner Bedienung nicht im Blick haben. An den Auftrag gebunden, soziale Versorgung intermediär zu bewerkstelligen, disponiert die organisierte Wohlfahrtspflege über die Mittel zu deren Ausgestaltung und trägt dafür mit den an ihr Beteiligten ökonomische Verantwortung. 